\title{
CORRECTION
}

\section{Author Correction: SARS-CoV-2 receptor binding domain radio-probe: a noninvasive approach for angiotensin- converting enzyme 2 mapping in mice}

Dan Li ${ }^{1}$, Jin Ding ${ }^{1}$, Te-li Liu ${ }^{1}$, Feng Wang ${ }^{1}$, Xiang-xi Meng ${ }^{1}$, Song Liu ${ }^{1}$, Zhi Yang ${ }^{1}$ and Hua Zhu ${ }^{1}$

Acta Pharmacologica Sinica (2022) 43:1885-1886; https://doi.org/10.1038/s41401-021-00848-5

Correction to: Acta Pharmacologica Sinica https://doi.org/10.1038/ s41401-021-00809-y, published online 23 November 2021

The authors apologized that the representative images of analysis of micro-PET imaging (Fig. 5a) appeared incorrectly. The title of the image at $24 \mathrm{~h}$ was written as $4 \mathrm{~h}$ accidentally. The correct images are presented. The authors declare that these corrections do not change the results or conclusions of this paper. The authors apologize for any inconvenience caused to the journal and readers.

\footnotetext{
'Key Laboratory of Carcinogenesis and Translational Research (Ministry of Education/Beijing), NMPA Key Laboratory for Research and Evaluation of Radiopharmaceuticals (National Medical Products Administration), Department of Nuclear Medicine, Peking University Cancer Hospital \& Institute, Beijing 100142, China

Correspondence: Zhi Yang (pekyz@163.com) or Hua Zhu (zhuhuananjing@163.com)

These authors contributed equally: Dan Li, Jin Ding
}

Published online: 3 February 2022 


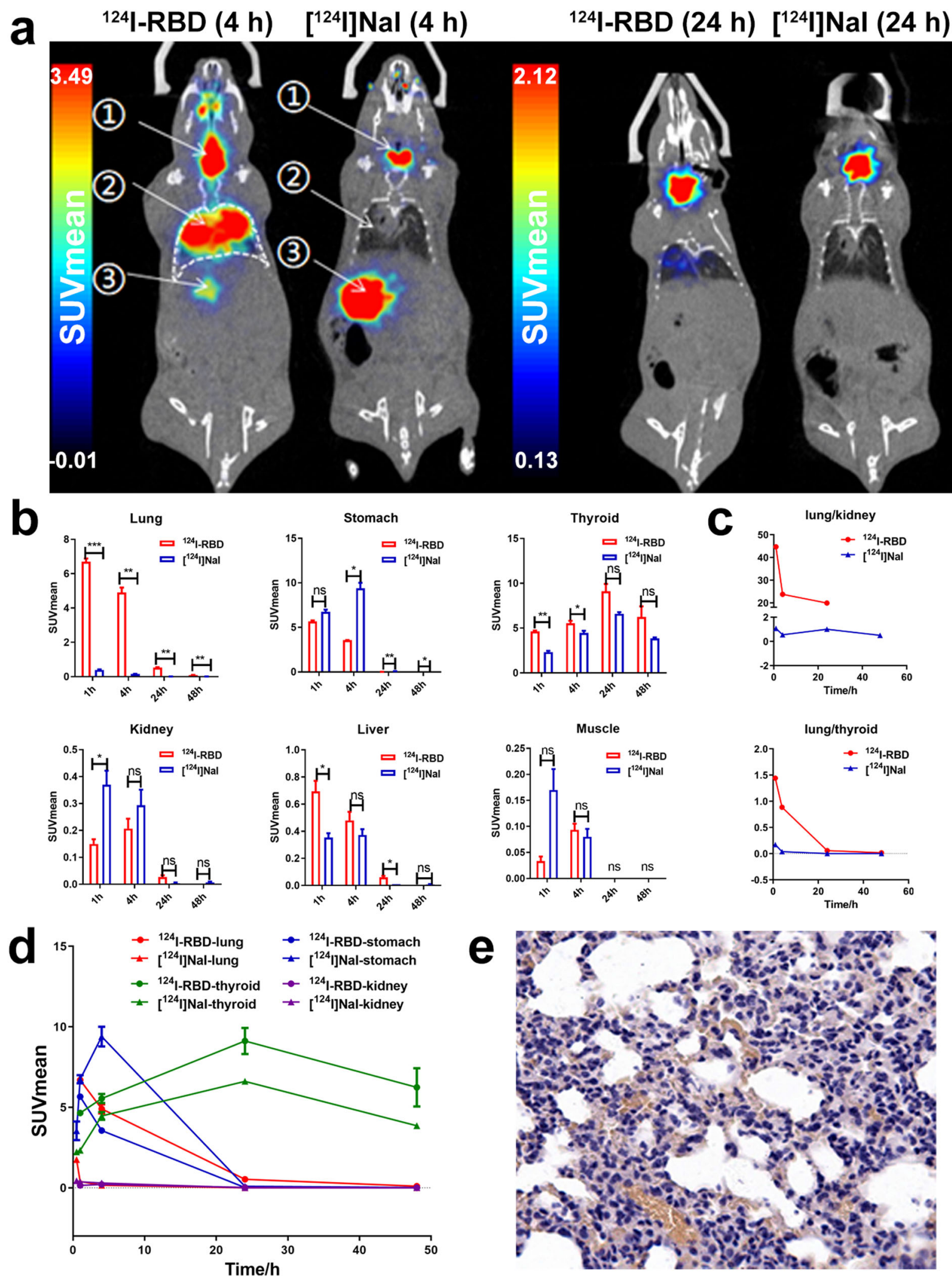

Fig. 5 Analysis of micro-PET imaging. a Slice images of lung uptake at 4 and $24 \mathrm{~h}$ after intrapulmonary injection of ${ }^{124} \mathrm{I}-\mathrm{RBD}$ and $\left.\mathrm{control}^{124} \mathrm{I}\right]$ Nal. (1), 2, (3) represent thyroid, lung and stomach of mice, respectively. b The SUV changes of ${ }^{124}$ I-RBD in lung, stomach, thyroid, kidney, liver, muscle and other organs as well as the control $\left[^{124} I\right]$ Nal at different time points. c Ratio of lung SUV to kidney and thyroid at different time points of ${ }^{124}$ I-RBD and control [ $\left.{ }^{124} \mathrm{I}\right] \mathrm{Nal}$. d The SUV of ${ }^{124} \mathrm{I}$-RBD and control $\left[{ }^{124} \mathrm{I}\right.$ INal in vital organs over time. Small animal PET/CT imaging was performed at 1, 4, 24 and $48 \mathrm{~h}$ after injection of ${ }^{124} \mathrm{I}-\mathrm{RBD}$ in mice, and $\left[{ }^{124} \mathrm{I}\right] \mathrm{Nal}$ increased the imaging time by $0.5 \mathrm{~h}$. e Immunohistochemistry of mouse lung tissue showed the expression of ACE2 in the lungs. 